\title{
Extension of explicit formulas in Poissonian white noise analysis using harmonic analysis on configuration spaces
}

\author{
Yu.G.Kondratiev ${ }^{1,2,3 *}$, T.Kuna $^{1,2 \dagger}$, M.J.Oliveira ${ }^{2,4,5 \ddagger}$ \\ ${ }^{1}$ Fakultät für Mathematik, Universität Bielefeld, D 33615 Bielefeld, Germany \\ 2 Forschungszentrum BiBoS, Universität Bielefeld, D 33615 Bielefeld, Germany \\ 3 National University "Kyiv-Mohyla Academy", Kiev, Ukraine \\ 4 Universidade Aberta, P 1269-001 Lisbon, Portugal \\ ${ }^{5}$ Centro de Matemática e Aplicações Fundamentais, University of Lisbon, P 1649-003 Lisbon, Portugal
}

Received January 31, 2008

\begin{abstract}
Harmonic analysis on configuration spaces is used in order to extend explicit expressions for the images of creation, annihilation, and second quantization operators in $L^{2}$-spaces with respect to Poisson point processes to a set of functions larger than the space obtained by directly using chaos expansion. This permits, in particular, to derive an explicit expression for the generator of the second quantization of a sub-Markovian contraction semigroup on a set of functions which forms a core of the generator.
\end{abstract}

Key words: Poisson measures, chaos decomposition, configuration spaces, annihilation operators, creation operators, second quantization operators

PACS: 02.40.Vh, 02.40.Yy

\section{Introduction}

This work is based on the previous papers $[4,6]$ and complements the results presented therein concerning the relation between Poissonian white noise analysis and the combinatorial harmonic analysis on configuration spaces. As it has been shown in [4], both analyses are related. In this work, we widen these relations towards annihilation and creation operators, and the second quantization operators as well.

In order to introduce the framework and the notation used throughout this work, in section 2 we recall the structure and concepts of Poissonian white noise analysis presented in [5] as well as the main notions and results of combinatorial harmonic analysis on configuration spaces presented in $[4,10]$. Within combinatorial harmonic analysis framework, in sections 3 and 4 we described the annihilation, creation, and second quantization operators defined on the Lebesgue-Poisson and Poisson spaces. The annihilation and creation operators are well-known in both spaces, see e.g., $[8,11]$ for the Poissonian case and $[3]$ for the Lebesgue-Poissonian one. Through the localization of the support of the Poisson measures, explicit expressions for these operators on the Poisson space can be given. The relation between the Poissonian annihilation and creation operators and the Lebesgue-Poissonian ones is given in section 3. In section 4 we give an explicit formula for the second quantization operator on the Poisson space stated in [1]. The interplay between combinatorial harmonic analysis and Poissonian white noise analysis allows us to derive this expression on a core of the second quantization operator. This is not obtainable in the framework of second quantization alone.

\footnotetext{
*E-mail: kondrat@mathematik.uni-bielefeld.de

${ }^{\dagger}$ E-mail: tkuna@mathematik.uni-bielefeld.de

‡E-mail: oliveira@cii.fc.ul.pt
} 


\section{Framework}

Throughout this work $X$ indicates a geodesically complete connected oriented (non-compact) $C^{\infty}$-Riemannian manifold, describing the position space of the particles. In particular, $X$ can be any $d$-dimensional Euclidean space $\mathbb{R}^{d}$, for $d \in \mathbb{N}$. There exists a sequence of open relative compact sets $\Lambda_{n}$ such that $\Lambda_{n} \subset \Lambda_{n+1}, n \in \mathbb{N}$, and $\bigcup_{n \in \mathbb{N}} \Lambda_{n}=X$.

\subsection{Configuration spaces and Poisson measures}

The configuration space $\Gamma:=\Gamma_{X}$ over $X$ is defined as the set of all locally finite subsets of $X$,

$$
\Gamma:=\{\gamma \subset X:|\gamma \cap K|<\infty \text { for every compact } K \subset X\},
$$

where $|\cdot|$ denotes the cardinality of a set. As usual we identify each $\gamma \in \Gamma$ with the non-negative Radon measure $\sum_{x \in \gamma} \delta_{x} \in \mathcal{M}(X)$, where $\delta_{x}$ is the Dirac measure with mass at $x, \sum_{x \in \emptyset} \delta_{x}$ is, by definition, the zero measure, and $\mathcal{M}(X)$ denotes the space of all non-negative Radon measures on the Borel $\sigma$-algebra $\mathcal{B}(X)$ on $X$. This procedure permits to endow $\Gamma$ with the topology induced by the vague topology on $\mathcal{M}(X)$. We denote the corresponding Borel $\sigma$-algebra on $\Gamma$ by $\mathcal{B}(\Gamma)$.

Let $\sigma$ be a non-atomic and non-degenerated Radon measure, i.e., $\sigma(\{x\})=0$ for all $x \in X$ and $\sigma(O)>0$ for all non-empty open sets $O \subset X$. Technically, the more challenging case is $\sigma(X)=\infty$. We define the Poisson measure $\pi_{\sigma}$ with intensity $\sigma$ as the unique probability measure on $(\Gamma, \mathcal{B}(\Gamma))$ with respect to which the following equality holds

$$
\int_{\Gamma} \mathrm{d} \pi_{\sigma}(\gamma) \exp \left(\sum_{x \in \gamma} \varphi(x)\right)=\exp \left(\int_{X} \mathrm{~d} \sigma(x)\left(\mathrm{e}^{\varphi(x)}-1\right)\right)
$$

for all $\varphi \in \mathcal{D}$. Here $\mathcal{D}:=\mathcal{D}(X)$ denotes the Schwartz space of all real-valued $C^{\infty}$-functions on $X$ with compact support.

Remark 1 One may also introduce the Poisson measure through its Laplace transform. This approach yields, through the Minlos theorem, a probability measure $\pi_{\sigma}$ defined on $\left(\mathcal{D}^{\prime}, \mathcal{C}_{\sigma}\left(\mathcal{D}^{\prime}\right)\right)$, where $\mathcal{D}^{\prime}$ is the dual space of $\mathcal{D}$ with respect to the space of real-valued functions $L_{R e}^{2}(X, \sigma) \subset L^{2}(X, \sigma)^{1}$ and $\mathcal{C}_{\sigma}\left(\mathcal{D}^{\prime}\right)$ is the $\sigma$-algebra generated by the cylinder sets

$$
\left\{\omega \in \mathcal{D}^{\prime}:\left(\left\langle\omega, \varphi_{1}\right\rangle, \ldots,\left\langle\omega, \varphi_{n}\right\rangle\right) \in B\right\}, \quad \varphi_{i} \in \mathcal{D}, B \in \mathcal{B}(X), n \in \mathbb{N} .
$$

An additional analysis shows that this measure is actually supported on generalized functions of the form $\sum_{x \in \gamma} \delta_{x}, \gamma \in \Gamma$. Hence $\pi_{\sigma}$ can be considered as a measure on $\Gamma$. For more details see e.g. [5] as well as the references therein.

Let us now consider the space of finite configurations

$$
\Gamma_{0}:=\bigsqcup_{n=0}^{\infty} \Gamma^{(n)}
$$

where $\Gamma^{(n)}:=\Gamma_{X}^{(n)}:=\{\gamma \in \Gamma:|\gamma|=n\}$ for $n \in \mathbb{N}$ and $\Gamma^{(0)}:=\{\emptyset\}$. For $n \in \mathbb{N}$, there is a natural bijection between the space $\Gamma^{(n)}$ and the symmetrization $\widetilde{X^{n}} / S_{n}$ of the set $\widetilde{X^{n}}:=\left\{\left(x_{1}, \ldots, x_{n}\right) \in\right.$ $X^{n}: x_{i} \neq x_{j}$ if $\left.i \neq j\right\}$ under the permutation group $S_{n}$ over $\{1, \ldots, n\}$ acting on $\widetilde{X^{n}}$ by permuting the coordinate index. This bijection induces a metrizable topology on $\Gamma^{(n)}$ and we endow $\Gamma_{0}$ with the topology of disjoint union of topological spaces. By $\mathcal{B}\left(\Gamma^{(n)}\right)$ and $\mathcal{B}\left(\Gamma_{0}\right)$ we denote the corresponding Borel $\sigma$-algebras on $\Gamma^{(n)}$ and $\Gamma_{0}$, respectively. In order to construct a measure on $\left(\Gamma_{0}, \mathcal{B}\left(\Gamma_{0}\right)\right)$ let us consider the above introduced measure $\sigma$ on $X$ and the product measures $\sigma^{\otimes n}$ on $X^{n}$, for $n \in \mathbb{N}$. For each $n \in \mathbb{N}$, let us also consider the image measure $\sigma^{(n)}$ on $\Gamma^{(n)}$ of the measure

\footnotetext{
${ }^{1}$ Throughout this work all $L^{p}$-spaces, $p \geqslant 1$, consist of complex-valued functions. A subscript "Re" will be added to indicate the corresponding real space.
} 
$\sigma^{\otimes n}$ under the mapping $\widetilde{X^{n}} \ni\left(x_{1}, \ldots, x_{n}\right) \mapsto\left\{x_{1}, \ldots, x_{n}\right\} \in \Gamma^{(n)}$. For $n=0$ we set $\sigma^{(0)}(\{\emptyset\}):=1$. Then, we define the so-called Lebesgue-Poisson measure $\lambda_{\sigma}$ on $\Gamma_{0}$ (with intensity $\sigma$ ) by the sum of measures

$$
\lambda_{z}:=\sum_{n=0}^{\infty} \frac{1}{n !} \sigma^{(n)} .
$$

The space $L^{2}\left(\Gamma_{0}, \lambda_{\sigma}\right)$ can be regarded as a realization of the symmetric Fock space over $L^{2}(X, \sigma)$ (denoted by $\operatorname{Exp} L^{2}(X, \sigma)$ ) by a space of functions. To an element $\left(f^{(n)}\right)_{n=0}^{\infty} \in \operatorname{Exp} L^{2}(X, \sigma):=$ $\bigoplus_{n=0}^{\infty} \hat{L}^{2}\left(X^{n}, \sigma^{\otimes n}\right)$, where $\hat{L}^{2}\left(X^{n}, \sigma^{\otimes n}\right)$ denotes the subspace of $L^{2}\left(X^{n}, \sigma^{\otimes n}\right)$ of all symmetric functions, one associates the function $G \in L^{2}\left(\Gamma_{0}, \lambda_{\sigma}\right)$ given by

$$
G\left(\left\{x_{1}, \ldots, x_{n}\right\}\right):=n ! f^{(n)}\left(x_{1}, \ldots, x_{n}\right), \quad n \in \mathbb{N} .
$$

Such a correspondence defines a unitary isomorphism between the spaces $\operatorname{Exp} L^{2}(X, \sigma)$ and $L^{2}\left(\Gamma_{0}, \lambda_{\sigma}\right)$. In particular, the image of a Fock coherent state $e(f):=\left(\frac{f^{\otimes n}}{n !}\right)_{n=0}^{\infty}$ under this isomorphism is the (Lebesgue-Poisson) coherent state $e_{\lambda}(f): \Gamma_{0} \rightarrow \mathbb{C}$ defined for any $\mathcal{B}(X)$-measurable function $f$ by

$$
e_{\lambda}(f, \eta):=\prod_{x \in \eta} f(x), \quad \eta \in \Gamma_{0} .
$$

The unitary isomorphism between the symmetric Fock space and $L^{2}\left(\Gamma_{0}, \lambda_{\sigma}\right)$ provides natural operators on the space $L^{2}\left(\Gamma_{0}, \lambda_{\sigma}\right)$ by carrying over standard Fock space operators. In particular, the annihilation and the creation operators, which images in $L^{2}\left(\Gamma_{0}, \lambda_{\sigma}\right)$, are well-known, see e.g. [3],

$$
\left(a_{\lambda}^{-}(\varphi) G\right)(\eta):=\int_{X} \mathrm{~d} \sigma(x) G(\eta \cup\{x\}) \varphi(x), \quad \eta \in \Gamma_{0},
$$

and

$$
\left(a_{\lambda}^{+}(\varphi) G\right)(\eta):=\sum_{x \in \eta} G(\eta \backslash\{x\}) \varphi(x), \quad \lambda_{\sigma}-a . a . \eta \in \Gamma_{0} .
$$

For more details and proofs see e.g. [5] and the references therein.

\subsection{Some aspects of Poissonian white noise analysis}

The description of elements of the space $L^{2}\left(\Gamma, \pi_{\sigma}\right)$ by a corresponding chaos decomposition provides a unitary isomorphism between the spaces $L^{2}\left(\Gamma, \pi_{\sigma}\right)$ and $L^{2}\left(\Gamma_{0}, \lambda_{\sigma}\right)$. This fact is recalled here (see the presentation in [5] and the references therein for more details and proofs).

As we mentioned in subsection 2.1, the Poisson measure $\pi_{\sigma}$ can be either considered on $(\Gamma, \mathcal{B}(\Gamma))$, on $\mathcal{M}(X)$, or on $\mathcal{D}^{\prime}$, where, in contrast to $\Gamma$, the spaces $\mathcal{M}(X)$ and $\mathcal{D}^{\prime}$ are linear. One has $\Gamma \subset \mathcal{M}(X) \subset \mathcal{D}^{\prime}$, but $\pi_{\sigma}(\Gamma)=1$. In what follows we shall always keep in mind the embeddings $\Gamma \subset \mathcal{M}(X) \subset \mathcal{D}^{\prime}$.

Given a $-1<\varphi \in \mathcal{D}$ let us consider the coherent state $e_{\pi}(\varphi)$ defined by

$$
e_{\pi}(\varphi, \omega):=\exp \left(\langle\omega, \log (1+\varphi)\rangle-\int_{X} \sigma(x) \varphi(x)\right)
$$

for $\omega \in \mathcal{D}^{\prime}$. The holomorphy of $e_{\pi}(\cdot, \omega), \omega \in \mathcal{D}^{\prime}$, on a neighborhood of zero permits to consider its Taylor expansion which, by the Cauchy formula, the polarization identity, and the kernel theorem (see e.g. $[2,7,9]$ ), provides the decomposition

$$
e_{\pi}(\varphi, \omega)=\sum_{n=0}^{\infty} \frac{1}{n !}\left\langle C_{n}^{\sigma}(\omega), \varphi^{\otimes n}\right\rangle, \quad \omega \in \mathcal{D}^{\prime},
$$

where $C_{n}^{\sigma}: \mathcal{D}^{\prime} \rightarrow \mathcal{D}^{\prime \hat{\otimes} n}, n \in \mathbb{N}$, are the so-called generalized Charlier kernels, which are pointwisely defined. For an explicit formula see (11) below. For $\varphi^{(n)} \in \mathcal{D}_{\mathbb{C}}^{\hat{\otimes} n}, n \in \mathbb{N}\left(\mathcal{D}_{\mathbb{C}}:=\right.$ the complexification of the space $\mathcal{D}$ ), we define the corresponding smooth Charlier monomial of order $n$ by 
$\left\langle C_{n}^{\sigma}(\omega), \varphi^{(n)}\right\rangle, \omega \in \mathcal{D}^{\prime}$. These monomials are orthogonal in $L^{2}\left(\Gamma, \pi_{\sigma}\right)$, which permits the use of an approximation procedure to extend the class of smooth Charlier monomials to $\pi_{\sigma}$-a.s. defined measurable monomials $\left\langle C_{n}^{\sigma}, f^{(n)}\right\rangle$ with kernels $f^{(n)} \in \hat{L}^{2}\left(X^{n}, \sigma^{\otimes n}\right)$ in such a way that the orthogonality property still holds for this extension. Note that a priori it is not clear in which sense, if at all, expression (11) extends as well.

Let us now consider the space $\mathcal{P}\left(\mathcal{D}^{\prime}\right)$ of smooth continuous polynomials on $\mathcal{D}^{\prime}, \mathcal{P}\left(\mathcal{D}^{\prime}\right):=$ $\left\{\Phi: \Phi(\omega)=\sum_{n=0}^{N}\left\langle C_{n}^{\sigma}(\omega), \varphi^{(n)}\right\rangle, \varphi^{(n)} \in \mathcal{D}_{\mathbb{C}}^{\hat{\otimes} n}, \omega \in \mathcal{D}^{\prime}, N \in \mathbb{N}_{0}\right\}$. Since this space is densely embedded into $L^{2}\left(\Gamma, \pi_{\sigma}\right)\left[13\right.$, section 10, Theorem 1], it follows that for any $F \in L^{2}\left(\Gamma, \pi_{\sigma}\right)$ there exists a sequence $\left(f^{(n)}\right)_{n=0}^{\infty}, f^{(n)} \in \hat{L}^{2}\left(X^{n}, \sigma^{\otimes n}\right), \sum_{n=0}^{\infty} n !\left|f^{(n)}\right|_{L^{2}\left(X^{n}, \sigma^{\otimes n}\right)}^{2}<\infty$ such that

$$
F=\sum_{n=0}^{\infty}\left\langle C_{n}^{\sigma}, f^{(n)}\right\rangle
$$

and, moreover, by the orthogonality property, $\|F\|_{L^{2}\left(\Gamma, \pi_{\sigma}\right)}^{2}=\sum_{n=0}^{\infty} n !\left|f^{(n)}\right|_{L^{2}\left(X^{n}, \sigma^{\otimes n}\right)}^{2}$. And vice versa, any series of the form (4) with $f^{(n)} \in \hat{L}^{2}\left(X^{n}, \sigma^{\otimes n}\right)$ and $\sum_{n=0}^{\infty} n !\left|f^{(n)}\right|_{L^{2}\left(X^{n}, \sigma^{\otimes n}\right)}^{2}<\infty$ defines a function from $L^{2}\left(\Gamma, \pi_{\sigma}\right)$. As a result, through the so-called chaos decomposition (4) we have defined a unitary isomorphism between $L^{2}\left(\Gamma, \pi_{\sigma}\right)$ and the symmetric Fock space $\operatorname{Exp} L^{2}(X, \sigma)$,

$$
I_{\pi}\left(\sum_{n=0}^{\infty}\left\langle C_{n}^{\sigma}, f^{(n)}\right\rangle\right):=\left(f^{(n)}\right)_{n=0}^{\infty} .
$$

Using the identification of $\operatorname{Exp} L^{2}(X, \sigma)$ and $L^{2}\left(\Gamma_{0}, \lambda_{\sigma}\right)$, the chaos decomposition (4) provides a natural unitary isomorphism $I_{\lambda \pi}$ between the spaces $L^{2}\left(\Gamma_{0}, \lambda_{\sigma}\right)$ and $L^{2}\left(\Gamma, \pi_{\sigma}\right)$ :

$$
\begin{aligned}
I_{\lambda \pi}(G) & =\sum_{n=0}^{\infty}\left\langle C_{n}^{\sigma}, f^{(n)}\right\rangle, \\
f^{(n)}\left(x_{1}, \ldots, x_{n}\right) & :=\frac{1}{n !} G\left(\left\{x_{1}, \ldots, x_{n}\right\}\right), n \in \mathbb{N}, f^{(0)}:=G(\emptyset) .
\end{aligned}
$$

In addition, the chaos decomposition permits to extend the definition (3) of a coherent state $e_{\pi}(\varphi)$ to other classes of functions $\varphi$. For this purpose, we first observe that given a $-1<\varphi \in \mathcal{D}$, for each $\gamma \in \Gamma$ we can write (3) as

$$
e_{\pi}(\varphi, \gamma)=\exp \left(-\int_{X} \mathrm{~d} \sigma(x) \varphi(x)\right) \prod_{x \in \gamma}(1+\varphi(x)) .
$$

Using the chaos decomposition one can extend the definition of coherent state to functions $f \in$ $L^{2}(X, \sigma)$ by $e_{\pi}(f, \gamma):=\sum_{n=0}^{\infty} \frac{1}{n !}\left\langle C_{n}^{\sigma}(\gamma), f^{\otimes n}\right\rangle$, but we may lose the explicit formula (5). Indeed, if $f \notin L^{1}(X, \sigma)$, then (5) is obviously not well-defined. However, one can show that for any function $f \in L^{1}(X, \sigma)$, expression (5) is $\pi_{\sigma}$-a.e. well-defined, and that the corresponding expression for $f \in L^{1}(X, \sigma) \cap L^{2}(X, \sigma)$ coincides with the extension in the $L^{2}$-sense, cf. [4]. It is clear that the image of a coherent state $e_{\pi}(f)$ with $f \in L^{2}(X, \sigma)$ under the isomorphism $I_{\lambda \pi}^{-1}$ is the above introduced (Lebesgue-Poisson) coherent state $e_{\lambda}(f)$.

\subsection{Some aspects of combinatorial harmonic analysis}

Besides the unitary isomorphism $I_{\lambda \pi}$, the $K$-transform also maps functions on $\Gamma_{0}$ into functions on $\Gamma$. To define the $K$-transform, let us consider the space $B_{b s}\left(\Gamma_{0}\right)$ of all complex-valued bounded $\mathcal{B}\left(\Gamma_{0}\right)$-measurable functions $G$ with bounded support, i.e., $\left.G\right|_{\Gamma_{0} \backslash\left(\sqcup_{n=0}^{N} \Gamma_{\Lambda}^{(n)}\right)} \equiv 0$ for some set $\Lambda \subset X$ with compact closure, $N \in \mathbb{N}_{0}$. Here $\Gamma_{\Lambda}^{(n)}:=\Gamma^{(n)} \cap\{\eta \in \Gamma: \eta \subset \Lambda\}$. The $K$-transform of a function $G \in B_{b s}\left(\Gamma_{0}\right)$ is the mapping $K G: \Gamma \rightarrow \mathbb{C}$ defined at each $\gamma \in \Gamma$ by

$$
(K G)(\gamma):=\sum_{\substack{\eta \subset \gamma \\|\eta|<\infty}} G(\eta)
$$


Note that for every $G \in B_{b s}\left(\Gamma_{0}\right)$ the sum in (6) has only a finite number of summands different from zero and thus $K G$ is a well-defined function on $\Gamma$. In particular, for any $\varphi \in \mathcal{D}$, one finds

$$
\left(K e_{\lambda}(\varphi)\right)(\gamma)=\prod_{x \in \gamma}(1+\varphi(x))
$$

It has been shown in [4] that the $K$-transform is a linear isomorphism which inverse mapping is defined, for instance, on $\mathcal{P}\left(\mathcal{D}^{\prime}\right)$ by

$$
\left(K^{-1} F\right)(\eta):=\sum_{\xi \subset \eta}(-1)^{|\eta \backslash \xi|} F(\xi), \quad \eta \in \Gamma_{0} .
$$

Furthermore, it has also been shown in [4] that on $B_{b s}\left(\Gamma_{0}\right)$ the inequality $\|K G\|_{L^{1}\left(\Gamma, \pi_{\sigma}\right)} \leqslant\|G\|_{L^{1}\left(\Gamma_{0}, \lambda_{\sigma}\right)}$ holds, allowing an extension of the $K$-transform to a bounded operator $K: L^{1}\left(\Gamma_{0}, \lambda_{\sigma}\right) \rightarrow L^{1}\left(\Gamma, \pi_{\sigma}\right)$. For the extended operator the explicit form (6) still holds, now $\pi_{\sigma}$-a.e. Since $e_{\lambda}(f) \in L^{p}\left(\Gamma_{0}, \lambda_{\sigma}\right)$ whenever $f \in L^{p}(X, \sigma)$ for some $p \in \mathbb{N}$, this means, in particular, that

$$
\left(K e_{\lambda}(f)\right)(\gamma)=\prod_{x \in \gamma}(1+f(x)), \quad \pi_{\sigma}-a . a . \gamma \in \Gamma
$$

for all $f \in L^{1}(X, \sigma)$, cf. e.g. [4].

There is a relation between Poissonian white noise analysis and combinatorial harmonic analysis through an equality of operators involving the unitary isomorphism $I_{\lambda \pi}$ and the $K$-transform $[4,10]$. This equality is given by

$$
I_{\lambda \pi}=K D^{-1}
$$

on $L^{1}\left(\Gamma_{0}, \lambda_{2 \sigma}\right) \cap L^{2}\left(\Gamma_{0}, \lambda_{\sigma}\right)\left[4\right.$, Theorem 5.1], where for all $G \in L^{1}\left(\Gamma_{0}, \lambda_{2 \sigma}\right)$

$$
\left(D^{-1} G\right)(\eta):=\int_{\Gamma_{0}} \mathrm{~d} \lambda_{\sigma}(\xi)(-1)^{|\xi|} G(\eta \cup \xi), \quad \lambda_{\sigma}-a . a . \eta \in \Gamma_{0} .
$$

In detail, let us observe that for $G \in B_{b s}\left(\Gamma_{0}\right), D^{-1} G$ is a pointwisely well-defined function on $\Gamma_{0}$ such that $D^{-1} G \in B_{b s}\left(\Gamma_{0}\right)$. The operator $D^{-1}$ is then the inverse of the linear isomorphism $D: B_{b s}\left(\Gamma_{0}\right) \rightarrow B_{b s}\left(\Gamma_{0}\right)$ defined by

$$
(D G)(\eta):=\int_{\Gamma_{0}} \mathrm{~d} \lambda_{\sigma}(\xi) G(\eta \cup \xi), \quad \eta \in \Gamma_{0} .
$$

Moreover, the inequalities of norms $\|D G\|_{L^{1}\left(\Gamma_{0}, \lambda_{\sigma}\right)},\left\|D^{-1} G\right\|_{L^{1}\left(\Gamma_{0}, \lambda_{\sigma}\right)} \leqslant\|G\|_{L^{1}\left(\Gamma_{0}, \lambda_{2 \sigma}\right)}$ hold, which permit to extend $D$ and $D^{-1}$ to bounded linear operators defined on $L^{1}\left(\Gamma_{0}, \lambda_{2 \sigma}\right)$. The extended operators are defined by (10), now only for $\lambda_{\sigma}$-a.a. $\eta \in \Gamma_{0}$, and (9), respectively.

Implications of equality (8) on the study of Poissonian white noise analysis and combinatorial harmonic analysis as well may be found in $[4,6,10,12]$. In particular, equality (8) yields an explicit expression for the Charlier monomials introduced in subsection 2.2. For all $f^{(n)} \in L^{1}\left(X^{n}, \sigma^{\otimes n}\right)$ symmetric and for $\pi_{\sigma}$-a.a. $\gamma \in \Gamma$,

$$
\left\langle C_{n}^{\sigma}(\gamma), f^{(n)}\right\rangle=\sum_{k=0}^{n} \sum_{\left\{x_{1}, \ldots, x_{k}\right\} \subset \gamma} \frac{n !(-1)^{n-k}}{(n-k) !} \int_{X^{n-k}} f^{(n)}\left(x_{1}, \ldots, x_{k}, y_{1}, \ldots, y_{n-k}\right) \mathrm{d} \sigma^{\otimes n-k}\left(y_{1}, \ldots, y_{k}\right) .
$$

For more details and proofs see [4].

\section{Annihilation and creation operators}

For $\varphi \in \mathcal{D}$, the operators $a_{\lambda}^{-}(\varphi)$ and $a_{\lambda}^{+}(\varphi)$ defined by (1) and (2), respectively, map the space $B_{b s}\left(\Gamma_{0}\right)$ into itself. Transported via the $K$-transform, the annihilation and creation operators give rise to reasonable expressions. 
Proposition 2 For each $\varphi \in \mathcal{D}$ and every $F \in K\left(B_{b s}\left(\Gamma_{0}\right)\right)$, the following relations hold:

$$
\left(\left(K a_{\lambda}^{-}(\varphi) K^{-1}\right) F\right)(\gamma)=\int_{X} \mathrm{~d} \sigma(x)(F(\gamma \cup\{x\})-F(\gamma)) \varphi(x), \quad \gamma \in \Gamma,
$$

and

$$
\left(\left(K a_{\lambda}^{+}(\varphi) K^{-1}\right) F\right)(\gamma)=\sum_{x \in \gamma} F(\gamma \backslash\{x\}) \varphi(x), \quad \pi_{\sigma}-a . a . \gamma \in \Gamma .
$$

Remark 3 It has been shown in [4] that $K\left(B_{b s}\left(\Gamma_{0}\right)\right)=\mathcal{F P}_{b c}(\Gamma)$, where

$$
\mathcal{F P}_{b c}(\Gamma):=\left\{\sum_{n=0}^{N}\left\langle C_{n}^{\sigma}, f^{(n)}\right\rangle: f^{(n)} \in B_{b s}\left(X^{n}\right) \text { symmetric, } n=1, \ldots, N \in \mathbb{N}\right\} .
$$

Proof. Given a $\varphi$ and a $F$ under the above conditions, the definitions of the operators $a_{\lambda}^{-}(\varphi), K$, and $K^{-1}$ yield

$$
\begin{aligned}
\left(\left(K a_{\lambda}^{-}(\varphi) K^{-1}\right) F\right)(\gamma)= & \int_{X} \mathrm{~d} \sigma(x) \sum_{\substack{\eta \subset \gamma \\
|\eta|<\infty}}\left(K^{-1} F\right)(\eta \cup\{x\}) \varphi(x) \\
= & \int_{X} \mathrm{~d} \sigma(x) \sum_{\substack{\eta \subset \gamma \\
|\eta|<\infty}} \sum_{\xi \subset \eta}(-1)^{|\eta \backslash \xi|} F(\xi \cup\{x\}) \varphi(x) \\
& +\int_{X} \mathrm{~d} \sigma(x) \sum_{\substack{\eta \subset \gamma \\
|\eta|<\infty}} \sum_{\xi \subset \eta}(-1)^{|\eta \backslash \xi|+1} F(\xi) \varphi(x) \\
= & \int_{X} \mathrm{~d} \sigma(x) K\left(K^{-1} F(\cdot \cup\{x\})\right)(\gamma) \varphi(x)-\int_{X} \mathrm{~d} \sigma(x) K\left(K^{-1} F\right)(\gamma) \varphi(x) \\
= & \int_{X} \mathrm{~d} \sigma(x) F(\gamma \cup\{x\}) \varphi(x)-F(\gamma) \int_{X} \mathrm{~d} \sigma(x) \varphi(x),
\end{aligned}
$$

for all $\gamma \in \Gamma$. The second equality is obtained from the definitions of the operators $K$ and $a_{\lambda}^{+}(\varphi)$ :

$$
\begin{aligned}
& \left(\left(K a_{\lambda}^{+}(\varphi) K^{-1}\right) F\right)(\gamma)=\sum_{\substack{\eta \subset \gamma \\
|\eta|<\infty}}\left(a_{\lambda}^{+}(\varphi)\left(K^{-1} F\right)\right)(\eta)=\sum_{\substack{\eta \subset \gamma \\
|\eta|<\infty}} \sum_{x \in \eta}\left(K^{-1} F\right)(\eta \backslash\{x\}) \varphi(x) \\
& \quad=\sum_{x \in \gamma} \varphi(x) \sum_{\eta \Subset \gamma \backslash\{x\}}\left(K^{-1} F\right)(\eta)=\sum_{x \in \gamma} \varphi(x)\left(K\left(K^{-1} F\right)\right)(\gamma \backslash\{x\})=\sum_{x \in \gamma} \varphi(x) F(\gamma \backslash\{x\}),
\end{aligned}
$$

for $\pi_{\sigma}$-a.a. $\gamma \in \Gamma$.

Remark 4 The previous proposition shows that

$$
K a_{\lambda}^{-}(\varphi) K^{-1}=a_{\pi}^{-}(\varphi)
$$

and

$$
K a_{\lambda}^{+}(\varphi) K^{-1}=a_{\pi}^{+}(\varphi)+\int_{X} \mathrm{~d} \sigma(x) \varphi(x),
$$

where $a_{\pi}^{-}(\varphi)$ and $a_{\pi}^{+}(\varphi)$ are the so-called (Poissonian) annihilation and creation operators. These operators are defined as the image of the corresponding operators on the Lebesgue-Poisson space under the unitary isomorphism $I_{\lambda \pi}$, that is,

$$
\begin{aligned}
& \left(a_{\pi}^{-}(\varphi) F\right)(\gamma)=\int_{X} \mathrm{~d} \sigma(x)(F(\gamma \cup\{x\})-F(\gamma)) \varphi(x), \quad \gamma \in \Gamma, \\
& \left(a_{\pi}^{+}(\varphi) F\right)(\gamma)=\sum_{x \in \gamma} \varphi(x) F(\gamma \backslash\{x\})-F(\gamma) \int_{X} \mathrm{~d} \sigma(x) \varphi(x), \quad \pi_{\sigma}-a . a . \gamma \in \Gamma,
\end{aligned}
$$

for $F \in \mathcal{F P}_{b c}(\Gamma)$, see e.g. [5]. 


\section{Second quantization operators}

Given a contraction operator $B$ on $L^{2}(X, \sigma)$ one may define a contraction operator $\operatorname{Exp} B$ on the Fock space $\operatorname{Exp} L^{2}(X, \sigma)$ acting on coherent states $e(f), f \in L^{2}(\sigma)$, by $\operatorname{Exp} B(e(f))=e(B f)$. In particular, given a positive self-adjoint operator $A$ on $L^{2}(X, \sigma)$ and the contraction semigroup $\mathrm{e}^{-t A}, t \geqslant 0$, one can define a contraction semigroup $\operatorname{Exp}\left(\mathrm{e}^{-t A}\right)$ on $\operatorname{Exp} L^{2}(X, \sigma)$ in this way. The generator is the well-known second quantization operator corresponding to $A$. We denote it by $d \operatorname{Exp} A$. Through the unitary isomorphism between the Fock space and the space $L^{2}\left(\Gamma_{0}, \lambda_{\sigma}\right)$ one may then define the corresponding operator in $L^{2}\left(\Gamma_{0}, \lambda_{\sigma}\right)$. We denote the (Lebesgue-Poisson) second quantization operator corresponding to $A$ by $H_{A}^{\mathrm{LP}}$. The action of $H_{A}^{\mathrm{LP}}$ on coherent states is given by

$$
\left(H_{A}^{\mathrm{LP}} e_{\lambda}(f)\right)(\eta)=\sum_{x \in \eta}(A f)(x) e_{\lambda}(f, \eta \backslash\{x\}), \quad f \in D(A) .
$$

Remark 5 According to (2), whenever $\mathcal{D} \subset D(A)$ one may rewrite the action of $H_{A}^{\mathrm{LP}}$ on coherent states $e_{\lambda}(\varphi), \varphi \in \mathcal{D}$, by $H_{A}^{\mathrm{LP}} e_{\lambda}(\varphi)=a_{\lambda}^{+}(A \varphi) e_{\lambda}(\varphi)$. Moreover, $H_{A}^{\mathrm{LP}} e_{\lambda}(\varphi)=e^{a_{\lambda}^{+}(\varphi)}(A \varphi)$, cf. e.g. [5, subsection 6.4], namely, Theorem 6.19 therein.

Proposition 6 Let $(A, D(A))$ be an operator in $L^{2}(X, \sigma)$. Define

$$
D_{1}(A):=\left\{f \in D(A) \cap L^{1}(X, \sigma): A f \in L^{1}(X, \sigma)\right\} .
$$

Then for all $f \in D_{1}(A)$ we have

$$
\left(D H_{A}^{\mathrm{LP}} D^{-1}\right) e_{\lambda}(f)=\left(H_{A}^{\mathrm{LP}}+\int_{X} \mathrm{~d} \sigma(x)(A f)(x)\right) e_{\lambda}(f)
$$

and

$$
\left(\left(K H_{A}^{\mathrm{LP}} K^{-1}\right) e_{\pi}(f)\right)(\gamma)=\sum_{x \in \gamma}(A f)(x) e_{\pi}(f, \gamma \backslash\{x\}), \quad \gamma \in \Gamma
$$

Proof. In order to prove equality (12), the so-called Ruelle convolution or $*$-convolution on $\mathcal{B}\left(\Gamma_{0}\right)$ measurable functions shows to be very useful. We recall that given two $\mathcal{B}\left(\Gamma_{0}\right)$-measurable functions $G_{1}$ and $G_{2}$, the *-convolution $G_{1} * G_{2}$ is a $\mathcal{B}\left(\Gamma_{0}\right)$-measurable function defined by

$$
\left(G_{1} * G_{2}\right)(\eta):=\sum_{\xi \subset \eta} G_{1}(\xi) G_{2}(\eta \backslash \xi), \quad \eta \in \Gamma_{0} .
$$

One easily sees that if $G_{1}=\tilde{G}_{1} \lambda_{\sigma}$-a.s. and $G_{2}=\tilde{G}_{2} \lambda_{\sigma}$-a.s., then also $G_{1} * G_{2}=\tilde{G}_{1} * \tilde{G}_{2} \lambda_{\sigma}$-a.s. This means, in particular, that $\left(H_{A}^{\mathrm{LP}} e_{\lambda}(f)\right)(\eta)=\left(e_{\lambda}(f) *(A f)\right)(\eta)$ for any $\lambda_{\sigma}$-a.s. finite version of $f \in D_{1}(A)$. Taking into account that the space $L^{1}\left(\Gamma_{0}, \lambda_{\sigma}\right)$ endowed with this product has a structure of a commutative algebra and $D\left(G_{1} * G_{2}\right)=\left(D G_{1}\right) *\left(D G_{2}\right)$ for any $G_{1}, G_{2} \in L^{1}\left(\Gamma_{0}, \lambda_{2 \sigma}\right)$, then one derives from the definition of the operator $D$ on $L^{1}\left(\Gamma_{0}, \lambda_{2 \sigma}\right)$, (10), the following equalities:

$$
\begin{aligned}
\left(D H_{A}^{\mathrm{LP}} D^{-1}\right) e_{\lambda}(f) & =\exp \left(-\int_{X} \mathrm{~d} \sigma(x) f(x)\right)\left(D H_{A}^{\mathrm{LP}}\right) e_{\lambda}(f) \\
& =\exp \left(-\int_{X} \mathrm{~d} \sigma(x) f(x)\right) D\left(e_{\lambda}(f) *(A f)\right) \\
& =\exp \left(-\int_{X} \mathrm{~d} \sigma(x) f(x)\right)\left(D e_{\lambda}(f)\right) *(D(A f))=\left(e_{\lambda}(f) *(D(A f))\right)(\eta) .
\end{aligned}
$$

Using the definition of the $*$-convolution, it is easy to check that for $\lambda_{\sigma}$-a.a. $\eta \in \Gamma_{0}$ the latter expression is equal to

$$
\sum_{x \in \eta}(A f)(x) e_{\lambda}(f, \eta \backslash\{x\})+\left(\int_{X} \mathrm{~d} \sigma(x)(A f)(x)\right) e_{\lambda}(f, \eta) .
$$


In this way equality (12) is proved. Concerning equality (13), note that by the definition of the operator $K$, by the definition of a coherent state $e_{\pi}(f)$ with $f \in L^{2}(X, \sigma) \cap L^{1}(X, \sigma)$, and (7) one finds

$$
\begin{aligned}
K\left(H_{A}^{\mathrm{LP}} e_{\lambda}(f)\right)(\gamma) & =\sum_{\substack{\eta \subset \gamma \\
|\eta|<\infty}} \sum_{x \in \eta}(A f)(x) e_{\lambda}(f, \eta \backslash\{x\})=\sum_{x \in \gamma}(A f)(x) \sum_{\substack{\eta \subset \gamma \backslash\{x\} \\
|\eta|<\infty}} e_{\lambda}(f, \eta) \\
& =\sum_{x \in \gamma}(A f)(x)\left(K e_{\lambda}(f)\right)(\gamma \backslash\{x\})=\sum_{x \in \gamma}(A f)(x) \prod_{x \in \gamma \backslash\{x\}}(1+f(x)) \\
& =\exp \left(\int_{X} \mathrm{~d} \sigma(x) f(x)\right) \sum_{x \in \gamma}(A f)(x) e_{\pi}(f, \gamma \backslash\{x\}),
\end{aligned}
$$

with

$$
e_{\lambda}(f)=\exp \left(\int_{X} \mathrm{~d} \sigma(x) f(x)\right) K^{-1} e_{\pi}(f) .
$$

Denote by $N$ the set of all $\gamma$ for which the first equality in (14) does not hold. Hence $\pi_{\sigma}(N)=0$. However, for the first equality appearing in (15) we need that $\pi_{\sigma}(\{\gamma: \exists x \in \gamma$ s.t. $\gamma \backslash x\})=0$. This follows from the Mackey identity, which yields

$$
\pi_{\sigma}(\{\gamma: \exists x \in \gamma \text { s.t. } \gamma \backslash x\}) \leqslant \limsup _{n \uparrow \infty} \int_{\Lambda_{n}} \sigma(\mathrm{d} x) \pi_{\sigma}(N)=0,
$$

where $\Lambda_{n}, n \in \mathbb{N}$, is the sequence of open relative compact sets defined at the beginning of section 2 .

Let us now consider the second quantization operator $H_{A}^{\mathrm{P}}:=I_{\lambda \pi} H_{A}^{\mathrm{LP}} I_{\pi \lambda}$ on the Poisson space (see e.g. $[1,5]$ ). The symmetric bilinear form corresponding to the operator $H_{A}^{\mathrm{P}}$ has the representation (cf. [2])

$$
\begin{aligned}
\left(H_{A}^{\mathrm{P}} F, G\right)_{L^{2}\left(\pi_{\sigma}\right)} & =\int_{\Gamma} \mathrm{d} \pi_{\sigma}(\gamma)\left(\left(a_{\pi}^{-} F\right)(\gamma), A\left(\left(a_{\pi}^{-} G\right)(\gamma)\right)\right)_{L^{2}(X, \sigma)} \\
\left(a_{\pi}^{-} F\right)(\gamma, x) & :=F(\gamma \cup\{x\})-F(\gamma), \quad \gamma \in \Gamma, x \in X
\end{aligned}
$$

for all Charlier polynomials $F$ and $G$. Here $(\cdot, \cdot)_{L^{2}(X, \sigma)}$ denotes the inner product on $L^{2}(X, \sigma)$. By using Proposition 6 one may reobtain the explicit formula for $H_{A}^{\mathrm{P}}$ derived e.g. in [1].

Corollary 7 For each operator $A$ under the conditions of Proposition 6 and every $f \in D_{1}(A)$ we have

$$
\left(H_{A}^{\mathrm{P}} e_{\pi}(f)\right)(\gamma)=\sum_{x \in \gamma}(A f)(x) e_{\pi}(f, \gamma \backslash\{x\})-\left(\int_{X} \mathrm{~d} \sigma(x)(A f)(x)\right) e_{\pi}(f, \gamma)
$$

for $\pi_{\sigma}$-a.a. $\gamma \in \Gamma$.

Proof. From equality (12) one obtains

$$
\left(D^{-1} H_{A}^{\mathrm{LP}} D\right) e_{\lambda}(f)=\left(H_{A}^{\mathrm{LP}}-\int_{X} \mathrm{~d} \sigma(x)(A f)(x)\right) e_{\lambda}(f) .
$$

This fact combined with equality (8) yields

$$
\begin{aligned}
H_{A}^{\mathrm{P}} e_{\pi}(f) & =\left(K D^{-1} H_{A}^{\mathrm{LP}}\right) e_{\lambda}(f)=\exp \left(-\int_{X} \mathrm{~d} \sigma(x) f(x)\right) K\left(D^{-1} H_{A}^{\mathrm{LP}} D e_{\lambda}(f)\right) \\
& =\exp \left(-\int_{X} \mathrm{~d} \sigma(x) f(x)\right)\left(K\left(H_{A}^{\mathrm{LP}} e_{\lambda}(f)\right)-\left(\int_{X} \mathrm{~d} \sigma(x)(A f)(x)\right) K e_{\lambda}(f)\right) \\
& =\left(K H_{A}^{\mathrm{LP}} K^{-1}\right) e_{\pi}(f)-\left(\int_{X} \mathrm{~d} \sigma(x)(A f)(x)\right) e_{\pi}(f)
\end{aligned}
$$


and the proof follows by equality (13).

Formula (16) has only a reasonable sense if it holds on a domain of essential self-adjointness of $H_{A}^{\mathrm{P}}$. Otherwise, the latter operator is not characterized by this formula. Due to the general theory of second quantization, clearly $\left\{\sum_{n=0}^{N}\left\langle C_{n}^{\sigma}, f^{(n)}\right\rangle: f^{(n)} \in D(A)^{\hat{\otimes} n}\right\}$ is a domain of essential selfadjointness. If $D(A)$ is not contained in the space of Schwartz test functions $\mathcal{D}$, the above monomials have to be understood, at least at the beginning, as $L^{2}$-extension, for which one does not have an explicit expression in general. To obtain an explicit expression, one has carefully to study in which sense and to which functions the explicit expression may be extended. For simplicity, in this section we have worked with coherent states. However, all results might be extended to monomials as well.

Corollary 8 If $(A, D(A))$ is an essentially self-adjoint operator and its closure generates a subMarkovian contraction semigroup, then $D_{1}(A)$ is a core of the generator of the $L^{1}$ - and of the $L^{2}$-semigroup. Then, also $\left\{e_{\pi}(f): f \in D_{1}(A)\right\}$ is a domain of essential self-adjointness of $H_{A}^{\mathrm{P}}$. Hence formula (16) holds on a domain of essential self-adjointness.

\section{Acknowledgements}

This work was supported by DFG through SFB-701 (Bielefeld University), by "Quantum Infinite Particle Systems in a Functional Integral Approach" (436 POL 113/115/0-1), and by FCT POCI, PDCT, and PTDC, FEDER.

\section{References}

1. Albeverio S., Kondratiev Yu.G., Röckner M. Analysis and geometry on configuration spaces. J. Funct. Anal., 1998, 154, No. 2, 444-500.

2. Berezansky Yu.M., Kondratiev Yu.G. Spectral Methods in Infinite-Dimensional Analysis. Naukova Dumka, Kiev, 1988. (in Russian). English translation, Kluwer Academic Publishers, Dordrecht, 1995.

3. Fichtner K.-H., Winkler G. Generalized Brownian motion, point processes and stochastic calculus for random fields. Math. Nachr., 1993, 161, 291-307.

4. Kondratiev Yu.G., Kuna T. Harmonic analysis on configuration space I. General theory. Infin. Dimens. Anal. Quantum Probab. Relat. Top., 2002, 5, No. 2, 201-233.

5. Kondratiev Yu.G., Kuna T., Oliveira M.J. Analytic aspects of Poissonian white noise analysis. Methods Funct. Anal. Topology, 2002, 8, No. 4, 15-48.

6. Kondratiev Yu.G., Kuna T., Oliveira M.J. On the relations between Poissonian white noise analysis and harmonic analysis on configuration spaces. J. Funct. Anal., 2004, 213, No. 1, 1-30.

7. Kondratiev Yu.G., Silva J.L., Streit L. Generalized Appell systems. Methods Functional Analysis and Topology, 1997, 3, No. 2, 28-61.

8. Kondratiev Yu.G., Silva J.L., Streit L., Us G.F. Analysis on Poisson and Gamma spaces. Infin. Dimens. Anal. Quantum Probab. Relat. Top., 1998, 1, No. 1, 91-117.

9. Kondratiev Yu.G., Streit L., Westerkamp W., Yan J. Generalized functions in infinite dimensional analysis. Hiroshima Math. J., 1998, 28, No. 2, 213-260.

10. Kuna T. Studies in Configuration Space Analysis and Applications. PhD thesis, Bonner Mathematische Schriften Nr. 324, University of Bonn, 1999.

11. Nualart D., Vives J. A duality formula on the Poisson space and some applications. In Seminar on Stochastic Analysis, Random Fields and Applications, 1995, 36, 205-213. Birkhäuser, Basel.

12. Oliveira M.J. Configuration Space Analysis and Poissonian White Noise Analysis. PhD thesis, Faculty of Sciences, University of Lisbon, 2002.

13. Skorohod A.V. Integration in Hilbert Space. Springer Verlag, Berlin, Heidelberg, and New York, 1974. 


\title{
Розширення явних формул аналізу Пуассона білого шуму за допомогою гармонічного аналізу на конфігураційних просторах
}

\author{
Ю.Г.Кондратьєв ${ }^{1,2,3}$, Т.Куна ${ }^{1,2}$, М.Ж.Олівейра ${ }^{2,4,5}$ \\ 1 Факультет математики, Університет Білефельда, Німеччина \\ 2 Дослідницький центр BiBoS, Університет Білефельда, Німеччина \\ 3 Національний університет “Києво-Могилянська академія”, Київ, Україна \\ 4 університет Аберта, Лісабон, Португалія \\ 5 Центр прикладних математичних досліджень, Університет Лісабону, Португалія
}

Отримано 31 січня 2008 р.

\begin{abstract}
За допомогою гармонічного аналізу на конфігураційних просторах одержано розширення явних виразів для образів операторів народження, знищення і вторинного квантування в $L^{2}$-просторах відносно точкових процесів Пуассона на клас функцій, ширший ніж простір одержаний безпосередньо з розкладу хаосу. Це дозволяє, зокрема, одержати явний вираз для генератора вторинного квантування під-марківської стискаючої півгрупи на множині функцій, які утворюють його ядро.
\end{abstract}

Ключові слова: міри Пуассона, декомпозиція хаосу, конфігураційні простори, оператори анігіляції, оператори створення, оператори вторинного квантування

PACS: $02.40 . \mathrm{Vh}, 02.40 . \mathrm{Yy}$ 